\title{
ON A FAMILY OF SIMPLE ORDERED GROUPS
}

\author{
VLASTIMIL DLAB \\ (Received 5 May 1967)
}

\section{Introduction}

In the present paper we shall consider some subgroups of (increasing) autohcmeomorphisms of the closed real interval $\langle 0 ; 1\rangle$; mainly because of brevity, we shall defer discussing possible generalizations of our results to more general ordered fields.

Groups of (order) automorphisms of the real line, or more generally, of some ordered sets have been frequently used in constructions of groups with some specified properties (cf., e.g., [1], [4], [5]); in particular, the group of all piecewise linear automorphisms of $\langle 0,1\rangle$ coinciding with the identical mapping in some neighbourhoods of 0 and 1 has served Chehata [1] in establishing the existence of an (algebraically) simple (linearly) ordered group.

Here, we present a whole family of (algebraically) simple (linearly) ordered groups $G_{H}$ : for every subgroup $H$ of the multiplicative group $R$ of all positive real numbers, $G_{H}$ is the group of all so-called locally right $H$-linear automorphisms of $\langle 0,1\rangle$ coinciding with the identical mapping in some neighbourhoods of 0 and 1 . In particular, $G_{H}$ is divisible if and only if $H$ is divisible; in fact, $G_{H}$ is then, in a certain sense, strongly divisible. There is a one-to-one correspondence between the (linear) orders of $G_{H}$ and those of $H$; thus, $G_{H}$ admits only two (linear) orders if and only if $H$ is of rank 1. Furthermore, $G_{R}$ contains an isomorphic copy of any (linearly) ordered abelian group of countable "Archimedean rank" and the continuum of non-order-isomorphic (linearly) ordered free groups of rank 2.

A particular value of our approach rests on the fact that simplicity and other properties of the groups $G_{H}$ can be established very easily.

\section{Preliminaries}

Let $G$ be the group of all increasing autohomeomorphisms of the interval $\langle 0,1\rangle$, i.e. the group of all continuous (strictly) increasing real functions on $\langle 0,1\rangle$ having 0 and 1 as their fixed points. Throughout the paper, they will be denoted by small Greek letters and written on the right: 


$$
x(\alpha \beta)=(x \alpha) \beta \text { for all } x \in\langle 0,1\rangle \text {; }
$$

$\varepsilon$ will always denote the unity of $G$.

An open interval $(a, b) \subseteq\langle 0,1\rangle$ is said to be a supporting interval of $\alpha \in G$ if $a \alpha=a, b \alpha \rightleftharpoons b$ and $x \alpha \neq x$ for all $x \in(a, b)$. The (at most countable disjoint) union of all supporting intervals of $\alpha$ is called the support of $\alpha$. An element of $G$ with a single supporting interval is said to be simple.

Let $H$ be a subgroup of the multiplicative group $R$ of all positive real numbers; in particular, $H_{r} \subset R$ denotes, for every $r \in R$, the least divisible subgroup of $R$ containing $r$. An automorphism $\alpha \in G$ is said to be $H$-linear in $\langle a, b\rangle \subseteq\langle 0,1\rangle$ if there is $h \in H$, such that

$$
x \alpha=a \alpha+h(x-a)_{1} \text { for all } x \in\langle a, b\rangle \text {; }
$$

$\infty$ is said to be piecerosse $H$-linear in $\langle a, b\rangle$ if there exists a finite number of $a_{\imath}$ 's: $a=a_{0} \nless a_{1}<\cdots<a_{n}=b$ such that $\alpha$ is $H$-linear in each $\left\langle a_{\imath+1}, a_{\imath}\right\rangle$, $1 \leqq i \leqq n$.

In what follows we shall consider the subgroup $\bar{G}_{H}$ of $G$ of the locally right $H$-linear automorphisms of $\langle 0,1\rangle$, i.e. the subgroup of all $\alpha \in G$ such that, for any $a \in\langle 0,1)$, there exists a positive real $e_{\alpha}^{a}$ and $h_{\alpha \alpha}^{a} \in H$ satisfying

$$
x \alpha=a \alpha+h_{\alpha}^{a}(x-a) \text { for all } x \in\left\langle a, a^{\prime}+e_{\alpha}^{a}\right\rangle ;
$$

thus, $\alpha \in \bar{G}_{H}$ if and only if, for any $a \in\langle 0,1)$, there is a non-trivial interval $\left\langle a, a+e_{\alpha}^{a}\right\rangle$ in which $\alpha$ is $H$-linear.

To every $\alpha \neq \varepsilon$ of $\bar{G}_{H}$, let us make to correspond the (at most countable) well-ordered subset $A_{\alpha}=\left\{a_{\alpha t}\right\}$ of $\langle 0,1\rangle$

$$
0 \leqq a_{\alpha 1}<a_{\alpha 2}<\cdots<a_{\alpha t}<\cdots \leqq 1
$$

defined as follows: $a_{\alpha t} \in A$ if and only if $a_{\alpha t} \alpha=a_{\alpha t}$ and there is no neighbourhood $N\left(a_{\alpha t}\right)$ of $a_{\alpha t}$ such that $x \alpha=x$ in $N\left(a_{\alpha t}\right)$. Notice that

$$
a_{\alpha \tau_{\alpha}}=\sup _{t} a_{\alpha t}
$$

is the greatest element of $A_{\alpha}$ and that $\alpha$ is simple if and only if $A_{\alpha}$ has two elements. In fact, $\left(a_{\alpha 1}, a_{\alpha 2}\right)$ is always a supporting interval of $\alpha$ and, furthermore, provided $a_{\alpha, t+2}$ exists, either $\left(a_{\alpha t}, a_{\alpha t+1}\right)$ or $\left(a_{\alpha_{t} t+1}, a_{\alpha t+2}\right)$ is a s'up'porting interval of $\alpha$.

Now, for every $\alpha \in \bar{G}_{H}$, consider the function $h_{\alpha}$ on $\langle 0,1)$ mapping each $a \in\langle 0,1)$ into the corresponding gradient $h_{\alpha}^{a} \in H$; as a matter of fact, we shall be particularly interested in the restriction of $h_{\alpha}$ to $A_{\alpha}$. Thus, $h_{\alpha}^{a_{\alpha t}} \neq 1$ if and only if $\left(a_{x t}, a_{\alpha t+1}\right)$ is a supporting interval of $\alpha$; of course, always $h_{\alpha}^{a_{\alpha_{1}}} \neq 1$. The function $h_{\alpha}$ restricted to $A_{\infty}$ describes what we like to call the basic characteristic of $\alpha$. More precisely, we shall say that $\alpha$ and $\beta$ of $\bar{G}_{H}$ have the same basic characteristic if there is an automorphism $\varphi \in G$ 'of 
$\langle 0,1\rangle$ (or, what appears to be the same, $\varphi \in \bar{G}_{H}$ or even $\varphi \in G_{H *}$ defined below) mapping $A_{\alpha}=\left\{a_{\alpha t}\right\}$ onto $A_{\beta}$ and satisfying

$$
h_{\alpha}^{a_{\alpha t}}=h_{\beta}^{a_{\alpha t} \phi} \text {. }
$$

Besides the subgroup $\bar{G}_{H}$ of $G$, we shall investigate the subgroups $G_{H *}$, $G_{* H}$ and $G_{H}$ of $\bar{G}_{H}: \alpha \neq \varepsilon$ belongs to $G_{H *}, G_{* H}$ or $G_{H}$ if and only if $0<a_{\alpha 1}$, $a_{\alpha \tau_{\alpha}}<1$ or $0<a_{\alpha 1}<a_{\alpha \tau_{\alpha}}<1$, respectively. Evidently, all three groups are normal in $\bar{G}_{H}$; in fact, $G_{H}$ is the only minimal normal subgroup of $\bar{G}_{H}$ (for the full normal structure of $\bar{G}_{H}$ see the diagram in Theorem 4.3.).

\section{Preparatory results}

Proposition 3.1. (cf. [5], [7]). Two non-unity elements $\alpha$ and $\beta$ of $\bar{G}_{H}, G_{H_{*}}, G_{* H}$ or $G_{H}$ are conjugate in the respective subgroup if and only if they have the same basic characteristic.

In particular, if $\alpha$ and $\beta$ of $\bar{G}_{H}$ have the same basic characteristic, then

$$
\beta=\Psi^{-1} \alpha \Psi
$$

with $\Psi \in G_{H}$ if $a_{\alpha \tau_{\alpha}}<1$ and $\Psi \in G_{H *}$ otherwise.

Proof. First, suppose that $\alpha$ and $\beta$ are conjugate in $G$, i.e. that

$$
\beta=\phi^{-1} \alpha \varphi \text { for a certain } \varphi \in G .
$$

Let $A_{\alpha}=\left\{a_{\alpha t}\right\}$. Then, evidently,

Moreover,

$$
A_{\beta}=\left\{a_{\beta t}\right\} \text {, where } a_{\beta t}=a_{\alpha t} \varphi \text {. }
$$

$$
h_{\beta}^{a_{\alpha t} \varphi}=h_{\varphi-\downarrow}^{a_{\alpha t} \varphi} h_{\alpha}^{a_{\alpha t}} h_{\varphi}^{a_{\alpha t}}=\left(h_{\varphi}^{a_{\alpha t}}\right)^{-1} h_{\alpha}^{a_{\alpha t}} h_{\varphi}^{a_{\alpha t}}=h_{\alpha}^{a_{\alpha t}},
$$

and thus, $\alpha$ and $\beta$ have the same basic characteristic.

Now, assume that $\alpha$ and $\beta$ have the same basic characteristic, i.e. that there is $\varphi \in G$ such that

$$
A_{\alpha}=\left\{a_{\alpha t}\right\}, A_{\beta}=\left\{a_{\alpha t} \beta\right\} \text { and } h_{\alpha}^{\alpha_{\alpha t}}=h_{\beta}^{a_{\alpha t} \varphi} .
$$

In order to construct an element $\Psi$ of $\bar{G}_{H}$ such that (3.1) holds, it is evidently sufficient to construct, for each supporting interval $\left(a_{\alpha t}, a_{\alpha t+1}\right)$ of $\alpha$, a function $\Psi_{t}$ of $G$ which is locally right $H$-linear in $\left\langle a_{\alpha t}, a_{\alpha t+1}\right\rangle$ and satisfies, moreover,

and

$$
a_{\alpha t} \Psi_{t}=a_{\alpha t} \varphi, \quad a_{\alpha t+1} \Psi_{t}=a_{\alpha t+1} \varphi
$$

$$
\Psi_{t}^{-1} \alpha \Psi_{t}=\beta \text { in }\left\langle a_{\alpha t} \varphi, a_{\alpha t+1} \varphi\right\rangle .
$$

For, then the continuous function $\Psi$ defined by

$$
x \Psi=x \Psi_{t} \text { in } \operatorname{each}\left(a_{\alpha t}, a_{\alpha+1}\right)
$$


and piecewise $H$-linear in each complementary interval of the support of $\alpha$ in $\langle 0,1\rangle$ belongs to $\bar{G}_{H}$ and satisfies (3.1); moreover, since our $\Psi_{1}$ will satisfy $x \Psi_{1}=x$ in a neighbourhood of $a_{\alpha 1}, \Psi$ can be taken from $G_{H}$ if $a_{\alpha} \tau_{\alpha}<1$ and from $G_{H *}$ otherwise.

Thus, to complete the proof, we are going to construct a function $\Psi_{t}$ with the appropriate properties. First, if $a_{\alpha t}>0$, let

$$
x \Psi_{t}=\left(a_{\alpha t} \varphi\right) a_{\alpha t}^{-1} x \text { in }\left\langle 0, a_{\alpha t}\right\rangle
$$

and, if $a_{\alpha t+1}<1$,

$$
x \Psi_{t}=1+\left(1-a_{\alpha t+1} \varphi\right)\left(1-a_{\alpha t+1}\right)^{-1} x \text { in }\left\langle a_{\alpha t+1}, 1\right\rangle .
$$

Without loss of generality, assume that

$$
h_{\alpha}^{a_{\alpha t}}>1 \text {. }
$$

Let $\alpha$ and $\beta$ be linear in $\left\langle a_{\alpha t}, a_{\alpha t}+e_{1}\right\rangle$ and $\left\langle a_{\alpha t} \varphi, a_{\alpha t} \varphi+e_{2}\right\rangle$, respectively. Take $e>0$ satisfying the relation

and define

$$
h_{\alpha}^{a_{x t}} \cdot e \leqq \min \left(e_{1}, e_{2}\right)
$$

Thus, for

$$
x \Psi_{t}=x+a_{\alpha t} \varphi-a_{\alpha t} \text { for } x \in\left\langle a_{\alpha t}, a_{\alpha t}+e\right\rangle .
$$

and

$$
\begin{aligned}
& x \in\left\langle a_{\alpha t} \varphi, a_{\alpha t} \varphi+e\right\rangle, \\
& x \Psi_{t}^{-1}=x+a_{\alpha t}-a_{\alpha t} \varphi
\end{aligned}
$$

$$
x\left(\Psi_{t}^{-1} \alpha \Psi_{t}\right)=\left[a_{\alpha t}+h_{\alpha}^{a_{\alpha t}}\left(x-a_{\alpha t} \varphi\right)\right] \Psi_{t}=x \beta .
$$

Now, consider the increasing sequence $\left\{a_{n}\right\}$ defined by

$$
a_{n}=\left(a_{\alpha, t}+e\right) x^{n-1} \text { for } n \geqq 1 .
$$

In view of $(3.2)$, we have

$$
\lim _{n} a_{n}=a_{x t^{+1}} .
$$

Hence, for every $x \in\left\langle a_{x t}+e, a_{\alpha_{t-1}}\right.$ ), there is (a unique) $n_{0} \geqq 1$ such that $a_{n_{0}}<x \leqq a_{n_{0}+1}$, and we complete the definition of $\Psi_{t}$ by putting

$$
x \Psi_{t}=x\left(\alpha^{-n_{0}} \Psi_{t} \beta^{n_{0}}\right)
$$

It is a matter of routine to check that $\Psi_{t}$ possesses all the required properties and thus to complete the proof of Proposition 3.1.

LEMMA 3.2. Let $\varepsilon \neq \omega \in \bar{G}_{H}$. If $a_{\omega 1}>0$, then-for any given $0 \leq a<a_{\omega 1}$, $a_{\omega \tau_{\omega}} \leqq b \leqq 1$ and $1 \neq h \in H$ - there are simple elements $\rho$ and $\sigma$ of $\bar{t}_{H}$ such that 
and

$$
a_{\rho 1}=a_{\sigma 1}=a, \quad h_{\rho}^{a}=h_{\sigma}^{a}=h, \quad a_{\rho \tau_{\rho}}=a_{\sigma \tau_{\sigma}}=b
$$

$$
\omega=\sigma^{-1} \rho .
$$

On the other hand, if $\varepsilon \neq \omega=\sigma^{-1} \rho$ with simple elements $\rho$ and $\sigma$ of $\bar{G}_{H}$ such that

then

$$
h_{\rho}^{a_{\rho 1}}=h_{\sigma}^{a_{\sigma_{1}}}=h, \quad h \neq h_{\omega}^{a_{\omega 1}}, \quad h \neq\left(h_{\omega}^{a_{\omega 1}}\right)^{-1},
$$

$$
0 \leqq a_{\rho 1}=a_{\sigma 1}<a_{\omega 1} \text { and } \max \left(a_{\rho \tau_{\rho}}, a_{\sigma \tau_{\sigma}}\right) \geqq a_{\omega \tau_{\omega}} .
$$

Proof. Define $\omega^{*} \in \bar{G}_{H}$ by

$$
x \omega^{*}=x \omega \text { for } x \omega \geqq x \text { and } x \omega^{*}=x \omega^{-1} \text { otherwise; }
$$

thus, $x \omega^{*} \geqq x$ for all $x \in\langle 0,1\rangle$. Put $h^{*}=\max \left(h, h^{-1}\right)$. Now, denote by $\sigma^{*}$ an element of $\bar{G}_{H}$ such that

$$
\begin{aligned}
& x \sigma^{*}=x \text { in }\langle 0, a\rangle, \\
& x \sigma^{*}=a+h^{*}(x-a) \text { in }\langle a, a+e\rangle \text { for } 0<e<a_{\omega 1}-a, \\
& x \omega^{*}<x \sigma^{*} \leqq b \text { in }\langle a+e, b\rangle
\end{aligned}
$$

and

$$
x \sigma^{*}=x \text { in }\langle b, 1\rangle .
$$

It is evident that such elements of $\bar{G}_{H}$ exist: Consider the greatest subintervals $\left\langle c_{1}, c_{2}\right\rangle$ of $\left\langle a_{\omega 1}, b\right\rangle$ such that $\omega^{*}$ is linear in $\left\langle c_{1}, c_{2}\right\rangle$; for each such interval put

$$
c_{i} \sigma^{*}=\frac{1}{2}\left(b+c_{i} \omega^{*}\right), \quad i=1,2,
$$

and $\sigma^{*}$ piecewise $H$-linear in $\left\langle c_{1}, c_{2}\right\rangle$; also, in $\left\langle a_{1}+e, a_{\omega 1}\right\rangle$, let $\sigma^{*}$ be piecewise $H$-linear with $(a+e) \sigma^{*}=a+h^{*} e$ and $a_{\omega 1} \sigma^{*}=\frac{1}{2}\left(b+a_{\omega 1}\right)$.

Now, if $h^{*}=h^{j}, j= \pm 1$, put $\sigma=\left(\sigma^{*}\right)^{j}$ and $\rho=\sigma \omega$. It is routine to check that $\beta$ satisfies all the requirements. The other part of Lemma 3.2 is obvious.

Proposition 3.3. Let $\alpha \neq \varepsilon$ be a non-simple element of $\bar{G}_{H}$. Then, there exists $\Psi \in \bar{G}_{H}$ such that

and

$$
\left.a_{\alpha 1}<a_{\Psi_{1}}, \quad a_{\Psi \tau_{\Psi}} \leqq a_{\alpha \tau_{x}} \quad \text { (i.e. } \Psi \in G_{H *}\right)
$$

satisfies

$$
\beta=\alpha \Psi^{-1} \alpha \Psi
$$

$$
A_{\beta}=\left\{a_{\alpha 1}, a_{\beta 2}, a_{\alpha \tau_{\alpha}}\right\} \text { with } h_{\beta}^{a_{x 1}}=\left(h_{\alpha}^{a_{x 1}}\right)^{2} .
$$

Moreover, given an arbitrary element $1+h \in H, \Psi$ can always be chosen so that

$$
h_{\beta}^{a_{2 \beta}}=h
$$


provided that two sequences $\left\{x_{n}\right\}$ and $\left\{y_{n}\right\}$ exist such that

and

$$
\lim _{n} x_{n}=\lim _{n} y_{n}=a_{\alpha \tau_{\alpha}}
$$

$$
x_{n} \alpha>x_{n}, \quad y_{n} \alpha<y_{n} \text { for } n \geqq 1 \text {. }
$$

Proof. Since the proof of the statement in each case to be considered has the same basic idea, we present here only the proof of the last assertion in the case $h_{\alpha}^{a_{\alpha}}<1$.

First, assume that $h>1$. Let

$$
a_{\alpha 1}<a_{\alpha 2} \leqq b_{1}<c_{1} \leqq \cdots \leqq b_{n}<c_{n} \leqq \cdots, \lim _{n} b_{n}=a_{\alpha \tau_{\alpha}}
$$

be a sequence of supporting intervals of $\alpha$ such that

$$
x_{\alpha}>x \text { in each }\left(b_{n}, c_{n}\right),
$$$$
n \geqq 1 \text {. }
$$

Define in each $\left(b_{n}, c_{n}\right)$ a subinterval $\left\langle u_{n}, v_{n}\right\rangle$ such that

$$
v_{n}<u_{n} \alpha
$$

and, moreover, such that $\alpha$ is linear in $\left\langle b_{1}, v_{1}\right\rangle$ and $u_{n}=v_{n}$ if, for $n \geqq 2$, $c_{n-1}=b_{n}$. Also, let $v_{0} \in\left(a_{\alpha 1}, a_{\alpha 2}\right)$ be a number such that $\alpha$ is linear in $\left\langle a_{\alpha 1}, v_{0}\right\rangle$; put $u_{0}=\frac{1}{2}\left(a_{\alpha 1}+v_{0}\right)$.

Let $\bar{\alpha} \in \bar{G}_{H}$ be defined by

and

$$
x \bar{\alpha}=x \alpha \text { for } x \in\left\langle a_{\alpha_{1}}, a_{\alpha 2}\right\rangle, x \in\left\langle b_{n}, c_{n}\right\rangle, \quad n \geqq 1
$$

$$
x \bar{\alpha}=x \text { otherwise. }
$$

Furthermore, define $\bar{\beta} \in \bar{G}_{H}$ in the following manner: with

(a) $\bar{\beta}$ is linear in $\left\langle a_{\alpha 1}, u_{0}\right\rangle,\left\langle u_{0}, w_{0}\right\rangle,\left\langle w_{0}, u_{1} \alpha^{-1}\right\rangle\left\langle u_{1} \alpha^{-1}, z_{0}\right\rangle$ and $\left\langle z_{0}, u_{1}\right\rangle$

$$
\begin{aligned}
& a_{\alpha_{1}} \bar{\beta}=a_{\alpha_{1}}, \quad\left(u_{1} \alpha^{-1}\right) \bar{\beta}=u_{1} \alpha^{-2}, \quad u_{1} \bar{\beta}=u_{1}, \\
& h_{\bar{\beta}}^{a_{\alpha 1}}=h_{\alpha}^{a_{\alpha_{1}}}, \quad h_{\bar{\beta}}^{u_{1} \alpha^{-1}}=h \cdot\left(h_{\alpha}^{b_{1}}\right)^{-1}, \\
& h_{\bar{\beta}}^{u_{0}} \leqq \frac{v_{0} \alpha-a_{\alpha 1}}{2\left(b_{1}-u_{0}\right)}, \quad h_{\bar{B}}^{u_{0}} \in H, \\
& h_{\bar{\beta}}^{w_{0}} \geqq \frac{u_{1} \alpha^{-2}-v_{0} \alpha}{u_{1} \alpha^{-1}-b_{1}}, \quad h_{\bar{\beta}}^{w_{0}} \in H,
\end{aligned}
$$

and

$$
h_{\bar{B}}^{z_{0}} \geqq \frac{h}{h-1}, \quad h_{\bar{B}}^{z_{0}} \in H .
$$

(b) for $n \geqq 1, \bar{\beta}$ is linear in $\left\langle v_{n}, w_{n}\right\rangle,\left\langle w_{n}, z_{n}\right\rangle$ and $\left\langle z_{n}, u_{n+1}\right\rangle$ with 


$$
\begin{aligned}
& w_{n}=\frac{1}{2}\left(v_{n}+c_{n}\right), \quad v_{n} \bar{\beta}=v_{n}, \quad u_{n+1} \bar{\beta}=u_{n+1}, \\
& h_{\bar{\beta}}^{v_{n}}=h_{\alpha}^{b_{n}}, \\
& h_{\bar{\beta}}^{w_{n}} \geqq \frac{b_{n+1}-w_{n}}{c_{n}-w_{n}}, \quad h_{\bar{\beta}}^{w_{n}} \in H,
\end{aligned}
$$

and

$$
h_{\bar{\beta}}^{z_{n}} \leqq \min \left(\frac{u_{n+1}-b_{n+1}}{u_{n+1}-c_{n}}, \frac{u_{n+1}-\left[v_{n}+h_{\alpha}^{b_{n}}\left(w_{n}-v_{n}\right)\right]}{u_{n+1}-w_{n}}\right), \quad h_{\bar{\beta}}^{z_{n}} \in H
$$

(c) $x \bar{\beta}=x$ otherwise.

Hence, $\bar{\alpha}$ and $\bar{\beta}$ have the same characteristic and therefore there is, in view of Proposition 3.1, $\Psi \in G_{H *}$ such that

$$
\bar{\beta}=\Psi^{-1} \bar{\alpha} \Psi
$$

Again, it is routine to check that $\beta=\alpha \Psi^{-1} \alpha \Psi$ has two supporting intervals $\left(a_{\alpha 1}, u_{1} \alpha^{-2}\right)$ and $\left(u_{1} \alpha^{-2}, a_{\alpha \tau_{\alpha}}\right)$ with

$$
h_{\beta}^{a_{\alpha 1}}=\left(h_{\alpha}^{a_{\alpha 1}}\right)^{2}<1 \text { and } h_{\beta}^{u_{1} \alpha^{-2}}=h>1 \text {. }
$$

Similarly, we deal with the case when $h<1$. Then, we assume that (3.3) satisfies

$$
x \alpha>x \text { in }\left(b_{1}, c_{1}\right) \text { and } x \propto<x \text { in each }\left(b_{n}, c_{n}\right), \quad n \geqq 2 \text {. }
$$

Again, we define $\bar{\beta}$ in a similar way as before with the exception of the interval $\left\langle a_{\alpha_{1}}, u_{2}\right\rangle$; there, $\bar{\beta}$ is linear in $\left\langle a_{\alpha_{1}}, u_{0}\right\rangle,\left\langle u_{0}, w_{0}\right\rangle,\left\langle w_{0}, u_{1} \alpha^{-1}\right\rangle,\left\langle u_{1} \alpha^{-1}, u_{1}\right)$, $\left\langle u_{1}, z_{0}\right\rangle,\left\langle z_{0}, u_{2}\right\rangle$ with

$$
\begin{gathered}
a_{\alpha 1} \bar{\beta}=a_{\alpha 1}, \quad\left(u_{1} \alpha^{-1}\right) \bar{\beta}=u_{1} \alpha^{-2}, \quad u_{2} \bar{\beta}=u_{2}, \\
h_{\bar{\beta}}^{a_{\alpha 1}}=h_{\alpha}^{a_{\alpha 1}}, \quad h_{\bar{\beta}}^{u_{1} \alpha^{-1}}=h \cdot\left(h_{\alpha}^{b_{1}}\right)^{-1},
\end{gathered}
$$

$h_{\bar{\beta}}^{u_{0}}, h_{\bar{\beta}}^{u_{1}} \in H$ sufficiently small and $h_{\bar{\beta}}^{w_{0}}, h_{\bar{\beta}}^{z_{0}} \in H$ sufficiently large. The proof can be then easily completed.

Proposition 3.4. (i) Let $\varepsilon \neq \omega \in G_{H}$. Then, for any $\varepsilon \neq \alpha \in \bar{G}_{H}$, there are $\kappa$ and $\lambda$ of $G_{H}$ such that

$$
\omega=\left(\kappa^{-1} \alpha \kappa\right)^{-1} \lambda^{-1} \alpha \lambda
$$

(ii) Let $\omega \in G_{H_{*}} \backslash G_{H}$. Then, for any $\alpha \in \bar{G}_{H} \backslash G_{* H}$, there are $\kappa, \lambda, \mu$ and $\nu$ of $G_{H_{*}}$ such that

$$
\omega=\left(\kappa^{-1} \alpha \kappa \mu^{-1} \alpha \mu\right)^{-1}\left(\lambda^{-1} \alpha \lambda \nu^{-1} \alpha \nu\right) .
$$

(iii) Let $\omega \in \bar{G}_{H} \backslash G_{H_{*}}$. Then $\omega$ belongs to the normal closure of $\alpha \in \bar{G}_{H}$ in $\bar{G}_{H}$ if and only if the cyclic subgroup of $R$ generated by $h_{\alpha}^{0}$ contains $h_{\omega}^{0}$ and, if $a_{\omega \tau_{\omega}}=1, a_{\alpha \tau_{\alpha}}=1$, as well. 
Proof. (i) This is an immediate consequence of Proposition 3.1 and Lemma 3.2. Indeed let $\bar{\alpha} \in \bar{G}_{H}$ be given by

$$
x \bar{\alpha}=x \alpha \text { for } x \in\left(a_{\alpha 1}, a_{\alpha 2}\right) \text { and } x \bar{\alpha}=x \text { otherwise. }
$$

Then, there are $\bar{\kappa}$ and $\overline{\hat{\lambda}}$ of $G_{H}$ such that $\omega=\sigma^{-1} \rho$ with

$$
\sigma=\bar{\kappa}^{-1} \bar{\alpha} \bar{\kappa}, \quad \rho=\bar{\lambda}^{-1} \bar{\alpha} \bar{\lambda} \quad \text { and } \quad a_{\sigma \tau_{\sigma}}=a_{\rho_{\tau_{\rho}}}=a_{\omega \tau_{\omega}}\left(=a_{\alpha 2} \bar{\kappa}=a_{\alpha 2} \bar{\lambda}\right) .
$$

Define $\kappa$ and $\lambda$ of $G_{H}$ to satisfy

$$
x \kappa=x \bar{\kappa} \text { and } x \bar{\lambda}=x \bar{\lambda} \text { for } x \in\left\langle 0, a_{\alpha 2}\right\rangle
$$

and to be identical and piecewise $H$-linear otherwise. Then

$$
\omega=\left(\kappa^{-1} \alpha \kappa\right)^{-1} \lambda^{-1} \alpha \lambda .
$$

(ii) First, according to Proposition 3.3, there is $\Psi \in G_{H_{*}}$ such that

$$
\bar{\alpha}=\alpha \Psi^{-1} \alpha \Psi
$$

has a supporting interval of the form $(a, 1)$. Then, Proposition 3.1 and Lemma 3.2 can be applied as in the previous case (i):

$$
\omega=\left(\bar{\kappa}^{-1} \bar{\alpha} \bar{\kappa}\right)^{-1} \bar{\lambda}^{-1} \bar{\alpha} \overline{\hat{\lambda}} ;
$$

finally, put $\kappa=\bar{\kappa}, \mu=\Psi \bar{\kappa}, \lambda=\bar{\lambda}, \nu=\Psi \bar{\lambda}$.

(iii) This part follows readily from Proposition 3.1.

EXAMPLE 3.5 As a matter of fact, very often we can make a stronger conclusion in the case (ii) similar to that of (i). However, the following example illustrates that, in general, such a conclusion does not hold:

Let $(a, 1), 0<a<1$, be a supporting interval of $\omega$ with $h_{\omega}^{a}>1$; let $x \alpha \geqq x$ for all $x \in\langle b, 1\rangle, 0<b<1$, and 1 be an accumulation point of the set of all $x$ 's such that $x \alpha=x$. Assume that, under these conditions, there are $\kappa$ and $\lambda$ of $\bar{G}_{R}$ such that

$$
\omega=\left(\kappa^{-1} \alpha \kappa\right)^{-1} \lambda^{-1} \alpha \lambda .
$$

First, there is evidently $0<c<1$ such that

Therefore, since

$$
c \alpha=c \text { and } c \lambda>\max (a, b \kappa) \text {. }
$$

$$
\begin{gathered}
x \kappa^{-1} \alpha \kappa \geqq x \text { for all } x \in\langle b \kappa, \mathbf{l}\rangle, \\
y=(c \lambda) \kappa^{-1} \alpha \kappa \geqq c \lambda>a .
\end{gathered}
$$

Thus, by a simple calculation, we get

$$
y \omega=\left[(c \lambda)\left(\kappa^{-1} \alpha \kappa\right)\right]\left(\kappa^{-1} \alpha \kappa\right)^{-1}\left(\lambda^{-1} \alpha \lambda\right)=c \alpha \lambda=c \lambda \leqq y,
$$

a contradiction of $h_{\omega}^{a}>1$. 
LEMMA 3.6. Let $\alpha \in \bar{G}_{H}$ be simple with the supporting interval $(a, b)$ and $h_{x}^{a}=h_{0}$; let $h \in H$. Then, there exists $\beta \in \bar{G}_{H}$, unique in $\bar{G}_{R}$, satisfying

(a) $\alpha \beta=\beta x$;

(b) $h_{\beta}^{a}=h$;

(c) each supporting interval of $\beta$ has a non-empty intersection with $(a, b)$.

If $h=1$, then $\beta=\varepsilon$; otherwise, $\beta$ is simple with the supporting interval $(a, b)$. Moreover, if

$$
h=h_{0}^{r} \text { for a rational } r=m / n, n>0,
$$

then $\beta$ is the (unique) solution of the equation

$$
\xi^{n}=\alpha^{m} \text {. }
$$

Proof. We shall sketch the proof in the case $h_{0}>1$; the basic idea is that of the proof of Proposition 3.1. Let $c>a$ be such that $\alpha$ is linear in $\langle a, c\rangle$ and take

put

$$
0<a_{1}<\min \left[c, a+h^{-1} h_{0}(c-a)\right]
$$

$$
a_{n}=a_{1} \alpha^{n-1} \text { for } n \geqq 1 \text {. }
$$

Evidently, $\lim _{n} a_{n}=b$ and, for every $x \in\left(a_{1}, b\right)$, there is a unique $n_{0} \geqq \mathbf{I}$ such that $a_{n_{0}}<x \leqq a_{n_{0}+1}$. Now, one can easily see that $\beta$ defined by

$$
\begin{array}{ll}
x \beta=a+h(x-a) \text { for } x \in\left\langle a, a_{1}\right\rangle, & \\
x \beta=x \alpha^{-n} \beta \alpha^{n} \text { for } x \in\left(a_{n}, a_{n+1}\right\rangle, & n \geqq 1,
\end{array}
$$

and

$$
x \beta=x \text { otherwise }
$$

satisfies (a), (b), (c) and is by these three properties uniquely determined. The rest of Lemma 3.6 follows easily.

Proposition 3.7. Let $\alpha \in \bar{G}_{H}$ and $r$ be a real number. Let $\left(h_{\alpha}^{a_{\alpha}}\right)^{r} \in H$ for every supporting interval $\left(a_{\alpha t}, a_{\alpha t+1}\right)$ of $\alpha$. Then there exists $\beta \in \bar{G}_{H}$, unique in $\bar{G}_{R}$, satisfying

(a) $\alpha \beta=\beta \alpha$;

(b) each supporting interval of $\beta$ intersects non-trivially some supporting interval of $\alpha$;

(c) for each $\left(a_{\alpha t}, a_{\alpha t+1}\right), h_{\beta}^{a_{\alpha} t}=\left(h_{\alpha}^{a_{\alpha} t}\right)^{r}$.

As a consequence, $\alpha$ and $\beta$ have the same supporting intervals. Also, if $r=m / n, n>0$, is rational, then $\beta=\alpha^{m / n}$ is the (unique) solution of the equation

$$
\xi^{n}=\alpha^{m}
$$


In general, for any $\alpha \in \bar{G}_{R}$ and any real $r$, a unique $\beta$ satisfying (a), (b), (c) always exists in $\bar{G}_{R}$; by definition, put

Then,

$$
\beta=\alpha^{r} \text {. }
$$

and

$$
\alpha^{r} \alpha^{s}=\alpha^{r+s}=\alpha^{8} \alpha^{r}
$$

$$
\left(\alpha^{r}\right)^{s}=\alpha^{r s}=\left(\alpha^{s}\right)^{r}
$$

for all real numbers $r$ and $s$.

Proof. The first part follows from the preceding Lemma 3.6 applied to each supporting interval of $\alpha$. But then we get readily the remaining relations for the "powers". For, $\beta=\alpha^{r} \alpha^{s}$ and $\beta=\alpha^{s} \alpha^{r}$ satisfy (a), (b) and (c) with

$$
h_{\beta}^{a_{\alpha} t}=h_{\alpha^{t}}^{a_{\alpha^{t}} t} h_{\alpha^{s}}^{a_{\alpha t} t}=h_{\alpha^{s}}^{a_{\alpha} t} h_{\alpha^{\tau}}^{a_{\alpha^{t}} t}=\left(h_{\alpha}^{a_{\alpha} t}\right)^{r+s} ;
$$

hence, in view of uniqueness,

$$
\alpha^{r} \alpha^{8}=\alpha^{8} \alpha^{r}=\alpha^{r+s} .
$$

Similarly, apply the first part of this Proposition 3.7 to $\alpha^{4}$ : Both $\beta_{1}=\left(\alpha^{r}\right)^{s}, \beta_{2}=\alpha^{r s}$ satisfy (i) and (ii); furthermore,

$$
h_{\beta_{1}}^{a_{\alpha t} t}=\left(h_{\alpha^{r}}^{a_{\alpha} t}\right)^{s}=\left[\left(h_{\alpha}^{a_{\alpha t}}\right)^{r}\right]^{s}=\left(h_{\alpha}^{a_{\alpha t} t}\right)^{r s}=h_{\beta_{2}}^{a_{\alpha t} t} .
$$

Hence, $\beta_{1}=\beta_{2}$, as required.

LemMA 3.8. (cf.[2]) Let

$$
W(\xi, \eta)=\xi^{k_{1}} \eta^{l_{1}} \xi^{k_{2}} \eta^{l_{\mathbf{2}}} \cdots \xi^{k_{n}} \eta^{l_{\mathbf{n}}}
$$

where all the integers $k_{i}, l_{i}(1 \leqq i \leqq n)$ with a possible exception of $l_{n}$ are non-zero, be a given "word". Let $0 \leqq a<b \leqq 1$. Then, for every $H \subseteq R$, there exist $\alpha$ and $\beta$ in $\bar{G}_{H}$ such that

and

$$
a_{\alpha 1}=a_{\beta 1}=a, \quad a_{\alpha \tau_{\alpha}}=a_{\beta \tau_{\beta}}=b
$$

$$
W(\alpha, \beta) \neq \varepsilon
$$

Proof. First, put

$$
k=\left|k_{1} l_{1} k_{2} l_{2} \cdots k_{n} l_{n}^{*}\right| \geqq 1,
$$

where $l_{n}^{*}=1$ if $l_{n}=0$ and $l_{n}^{*}=l_{n}$ otherwise. Choose $h_{0} \in H$ such that $h_{0}>3$. Furthermore, put

$$
h=h_{0}^{k}>3^{k} \geqq 3,
$$

and denote by $c$ a number satisfying $h \leqq c$; finally, choose

$$
0<e<\frac{b-a}{2 n c}
$$


put $a_{0}=a$ and

$$
a_{q}=a+q(h-1) e \text { for } 2 \leqq q \leqq 2 n-1 ;
$$

clearly, $a_{0}<a_{2}<\cdots<a_{2 n-1}<b$.

Now, in the interval $\left\langle a_{q}, a_{q}+2(h-1) e\right\rangle$ define the function $\Psi_{q}$ by

and

$$
\begin{aligned}
& x \Psi_{q}=a_{q}+h\left(x-a_{q}\right) \text { for } x \in\left\langle a_{q}, a_{q}+e\right\rangle, \\
& x \Psi_{q}=a_{q}+h e+h^{-1}\left(x-a_{q}-e\right) \text { for } x \in\left\langle a_{q}+e, a_{q}+e+h e\right\rangle
\end{aligned}
$$

$x \Psi_{q}=x$ otherwise.

By means of these functions then define $\bar{\alpha} \in \bar{G}_{H}$ and $\bar{\beta} \in \bar{G}_{H}$ in the following way:

and

(i) $x \bar{\alpha}=x$ in $\langle 0, a\rangle$ and $\langle b, 1\rangle$,

$x \bar{\alpha}=x \Psi_{2(i-1)}$ in $\left\langle a_{2(i-1)}, a_{2 i}\right\rangle$ for $1 \leqq i \leqq n-1$,

$x \bar{\alpha}=a_{2(n-1)}+h\left(x-a_{2(n-1)}\right)$ in $\left\langle a_{2(i-1)}, a_{2(i-1)}+e\right\rangle$

$x \bar{\alpha} \neq x, \bar{\alpha}$ piecewise $H$-linear otherwise.

(ii) $x \bar{\beta}=x$ in $\langle 0, a\rangle,\langle a+2 h e, a+3(h-1) e\rangle$ and $\langle b, 1\rangle$,

$x \bar{\beta}=x \Psi_{2 i-1}$ in $\left\langle a_{2 i-1}, a_{2 i+1}\right\rangle$ for $2 \leqq i \leqq n-1$,

$x \bar{\beta}=a+h(x-a)$ in $\langle a, a+e\rangle$,

$x \bar{\beta}=x+(h-1) e$ in $\langle a+e, a+h e\rangle$,

$x \bar{\beta}=a_{2 n-1}+h\left(x-a_{2 n-1}\right)$ in $\left\langle a_{2 n-1}, a_{2 n-1}+e\right)$

and

$x \bar{\beta} \neq x, \bar{\beta}$ piecewise $H$-linear otherwise.

Finally, apply Lemma 3.6, and define $\alpha \in \bar{G}_{H}$ by

$$
x \alpha=x \bar{\alpha}^{-k_{i}} \text { in }\left\langle a_{2(i-1)}, a_{2 i}\right\rangle \text { for } 1 \leqq i \leqq n-1
$$

and

$$
x \alpha=x \bar{\alpha}^{-k_{n}} \text { otherwise. }
$$

Similarly, take $\beta \in \bar{G}_{H}$ satisfying

and

$$
\begin{aligned}
& x \beta=x \bar{\beta}^{-l_{1}} \text { in }\left\langle a, a_{3}\right\rangle, \\
& x \beta=x \bar{\beta}^{-l_{i}} \text { in }\left\langle a_{2 i-1}, a_{2 i+1}\right\rangle \text { for } 2 \leqq i \leqq n-1
\end{aligned}
$$

$x \beta=x \bar{\beta}^{-l_{\boldsymbol{n}}^{*}}$ otherwise.

A routine calculation shows that

as required.

$$
(a+e) W(\alpha, \beta)=a+e+2 n e(h-1) \neq a+e,
$$




\section{Theorems}

In this final section, we shall derive - using the results of the preceding section - the main properties of the groups $G_{H}, H \cong R$. As a matter of fact, formulations of the results in $\S 3$ allow many statements on $G_{H}$ to be extended to $\bar{G}_{H}$ etc.

Theorem 4.1. For any subgroup $H \subseteq R, G_{H}$ can be linearly ordered. In fact, there is a one-to-one correspondence between the linear orders of $H$ and those of $G_{H}$. Hence, $G_{H}$ has only two linear orders if and only if $H$ is of rank 1, i.e. if and only if $H$ is a subgroup of $H_{r}$ for a suitable $r \in R$.

Proof. Let $P(H)$ be the positive cone of a linear order in $H$. Define $P\left(G_{H}\right)$ by

$$
\omega \in P\left(G_{H}\right) \text { if and only if } \omega=\varepsilon \text { or } h_{\omega}^{a_{\omega} 1} \in P(H) .
$$

In view of Proposition 3.1, $P\left(G_{H}\right)$ is the positive cone of a linear order of $G_{H}$.

On the other hand, let $P\left(G_{H}\right)$ be the positive cone of a linear order of $G_{H}$. Evidently, $P\left(G_{H}\right)$ contains simple elements: let $\sigma \in P\left(G_{H}\right)$ be simple, $(a, b)$ its supporting interval and $h_{\sigma}^{a}=h_{0}$. We are going to show that any $\omega \in G_{H}$ with $h_{\omega}^{a_{\omega 1}}=h_{0}$ belongs to $P\left(G_{H}\right)$. First, take $\Psi_{0} \in G_{H}$ such that

satisfies

$$
\beta=\sigma^{2} \Psi_{0}^{-1} \sigma \Psi_{0} \in P\left(G_{H}\right)
$$

$$
A_{\beta}=\{a, b, c\}, \quad h_{\beta}^{a}=h_{0}^{2}, \quad h_{\beta}^{b}=h_{0} .
$$

Now, given $1 \neq h \in H$, there are, according to Proposition 3.3, always three elements $\alpha, \Psi_{1}$ and $\Psi_{2}$ of $G_{H}$ such that

satisfy

$$
\beta_{1}=\alpha \Psi_{1}^{-1} \alpha \Psi_{1} \text { and } \beta_{2}=\alpha \Psi_{2}^{-1} \alpha \Psi_{2}
$$

and

$$
A_{\beta_{1}}=\{a, b, c\}, \quad h_{\beta_{1}}^{a}=h_{0}^{2}, \quad h_{\beta_{1}}^{a}=h_{0}
$$

$$
A_{\beta_{2}}=\{a, b, c\}, \quad h_{\beta_{2}}^{a}=h_{0}^{2}, \quad h_{\beta_{2}}^{a}=h .
$$

In view of Proposition 3.1, $\beta_{1} \in P\left(G_{H}\right)$ and thus, necessarily, $\alpha \in P\left(G_{H}\right)$. Hence, $\beta_{2} \in P\left(G_{H}\right)$. Since, again by Propositions 3.1 and 3.3, there is $\Psi \in G_{H}$ such that $\omega \Psi^{-1} \omega \Psi$ and $\beta_{2}$ are conjugate, we get $\omega \in P\left(G_{H}\right)$, as required.

From here we deduce immediately that the correspondence

$$
\begin{gathered}
\text { " } h \in H \text { belongs to } P(H) \text { if and only if all } \omega \in G_{H} \\
\text { with } h_{\omega}^{a_{\omega 1}}=h \text { belong to } P\left(G_{H}\right) "
\end{gathered}
$$

between the linear orders of the groups $H$ and $G_{H}$ is one-to-one.

The rest of Theorem 4.1 follows easily.

Another consequence of Proposition 3.3 is the following 
THEOREM 4.2. Let $F_{H} \subseteq G_{H}$ be the subgroup of all elements of $G_{H}$ which are piecewise $H$-linear (in $\langle 0,1\rangle$ ). Thus, for every $\varepsilon \neq \alpha \in F_{H}$, $\alpha$ is linear in an interval $\left\langle a, a_{\alpha \tau_{\alpha}}\right\rangle$; put $h_{\alpha}=h_{\alpha}^{a}$. Let $P(H)$ be the positive cone of a certain linear order of $H$. Define the linear order in $F_{H}$ by

$$
\varepsilon \neq \omega \in P\left(F_{H}\right) \text { if and only if } h_{\omega} \in P(H) \text {. }
$$

This (partial) order of $G_{H}$ cannot be extended to a linear order of $G_{H}$, i.e. $G_{H}$ is not an $O^{*}$-group in the sense of [3].

Proof. Again, given $h \in P(H)$, there are, by Propositions 3.1 and 3.3, $\alpha, \Psi_{1}$ and $\Psi_{2}$ of $G_{H}$ such that

belong to $F_{H}$ and

$$
\beta_{1}=\alpha \Psi_{1}^{-1} \alpha \Psi_{1} \text { and } \beta_{2}=\alpha \Psi_{2}^{-2} \alpha \Psi_{2}
$$

$$
h_{\beta_{1}}=h=\left(h_{\beta_{\mathbf{2}}}\right)^{-1} \text {. }
$$

Thus, $\beta_{1} \in P\left(F_{H}\right)$ whilst $\beta_{2} \notin P\left(F_{H}\right)$; consequently, there is no linear order of $G_{H}$ extending the given order of $F_{H}$.

THEOREM 4.3. For any subgroup $H \subseteq R, G_{H}$ is (algebraically) simple. In fact, the following diagram

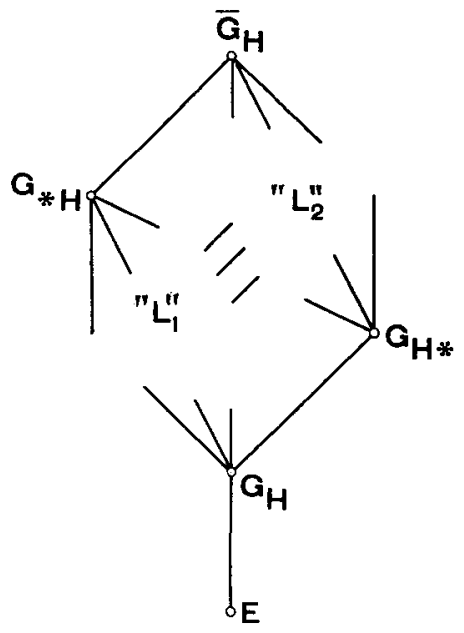

where $\boldsymbol{L}_{\mathbf{1}} \cong \boldsymbol{L}_{\mathbf{2}}$ are isomorphic to the lattice of all subgroups of $H$ describes the full normal structure of $\bar{G}_{H}\left(G_{* H} / G_{H} \cong \bar{G}_{H} / G_{H *} \cong H\right)$.

Proof. The first part of Theorem 4.3 follows immediately from Proposition 3.4 (i). As a matter of fact, the diagram is a straightforward consequence of Proposition 3.4, as well.

THEOREM 4.4. The group $G_{H}$ is divisible if and only if $H \subseteq R$ is divisible. In fact, $G_{H}$ is then strongly divisible in the following sense: Every equation 


$$
\xi^{n_{1}} \alpha_{1} \xi^{n_{2}} \alpha_{2} \cdots \xi^{n_{q}} \alpha_{q}=\varepsilon \text { with } n_{i}>0, \alpha_{i} \in G_{H} \text { for } 1 \leqq i \leqq q
$$

possesses a unique solution $\xi_{1}$ in $G_{H}$.

Also, $G_{R}$ is "continuously" divisible in the sense that every equation

$$
\xi^{r}=\alpha \text { with a real exponent } r \text { and } \alpha \in G_{R}
$$

has a (unique) solution in $G_{R}$.

Proof. Again, the first and the last parts of Theorem 4.4 follow immediately from Proposition 3.7.

However, the statement on solvability of (4.1) (in the case when $H$ is divisible) needs an independent proof: We shall construct a solution $\xi_{1}$ of (4.1) and - at the same time - show that it is unique. It is evident that, without loss of generality, we can assume $n_{i}=1$ for all $1 \leqq i \leqq q$. Moreover, throughout the proof we shall always denote the products

$$
\xi_{x} \alpha_{1} \xi_{x} \alpha_{2} \cdots \xi_{x} \alpha_{i-1}(1 \leqq i \leqq q) \text {, where } \xi_{x} \in G_{H},
$$

by $W_{i}\left(\xi_{x}\right)$; in particular, $W_{1}\left(\xi_{x}\right)=\varepsilon$.

Discarding the trivial case, assume that $\alpha_{i} \neq \varepsilon$ for at least one $i$ and put

$$
a=\min _{\alpha_{i} \neq \varepsilon}\left(a_{\alpha_{i}}\right)>0 .
$$

Notice that $\xi_{a}=\varepsilon$ satisfies (4.1) in the interval $\langle 0, a\rangle$; moreover, any $\xi \in G_{H}$ satisfying $(4.1)$ in $\langle 0, a\rangle$ is necessarily identical with $\varepsilon$ in this interval.

Let $N_{1}$ be the set of all $1 \leqq i \leqq q$ such that $a_{\alpha_{i} 1}=a$. Define $h_{a} \in H$ as the (unique) real number satisfying

$$
h_{a}=\prod_{i \in N_{1}}\left(h_{\alpha_{i}}^{a}\right)^{-1 / q} .
$$

Consider an element $\Psi_{1} \in G_{H}$ such that

and

$$
x \Psi_{1}=x \text { in }\langle 0, a\rangle
$$

$$
x \Psi_{1}=a+h_{a}(x-a) \text { in }\left\langle a, a+e_{1}\right\rangle \text { for a suitable } e_{1}>0 .
$$

Clearly, there is $b>a$ such that $\xi_{b}=\Psi$ satisfies $(4.1)$ in $\langle 0, b\rangle$ and

$$
(b \leqq) b^{*}=\max _{1 \leqq i \leqq q}\left[b W_{i}\left(\xi_{b}\right)\right] \leqq a+e_{1} .
$$

Moreover, if any other $\xi \in G_{H}$ satisfies $(4.1)$ in $\langle 0, b\rangle$, then $\xi$ necessarily coincides with $\xi_{b}$ in $\left\langle 0, b^{*}\right\rangle$.

Now, denote by $I$ the (non-empty) subset of $(0,1\rangle$ of all $x$ such that there is an element $\xi_{x} \in G_{H}$ satisfying (4.1) in $\langle 0, x\rangle$. Evidently, if $x_{1}<x_{2}$ and $x_{2} \in I$, then $x_{1} \in I$. Furthermore, for each $x \in I$ and the corresponding $\xi_{x}$ define, for every $z \in\langle 0, x\rangle$ 


$$
(z \leqq) z^{*}\left(\xi_{x}\right)=\max _{1 \leqq i \leqq q}\left[z W_{i}\left(\xi_{x}\right)\right]
$$

Notice that always $z^{*}\left(\xi_{x}\right)<1$ unless $z=x=1$ and that $z_{1}<z_{2}$ implies $z_{1}^{*}(\xi)<z_{2}^{*}(\xi)$.

First, we are going to show that, for $0<x_{1} \leqq x_{2}$, the corresponding functions $\xi_{x_{1}}$ and $\xi_{x_{2}}$ coincide in the interval $\left\langle 0, x_{1}^{*}\left(\xi_{x_{1}}\right)\right\rangle$. In order to prove the assertion, denote by $J$ the set of all $y \in\left\langle 0, x_{1}^{*}\left(\xi_{x_{1}}\right)\right\rangle$ such that

$$
x \xi_{x_{1}}=x \xi_{x_{2}} \text { for } x \in\langle 0, y\rangle \text {. }
$$

Certainly, since every $y \leqq \min \left(x_{1}^{*}\left(\xi_{x_{1}}\right), b\right)$ belongs to $J, J$ is non-empty. Also, in view of continuity of the functions $\xi_{x_{1}}$ and $\xi_{x_{2}}$, we get necessarily

$$
\sup _{y \in J} y=y_{0} \in J \text {. }
$$

We claim that $y_{0}=x_{1}^{*}\left(\xi_{x_{1}}\right)$. Assuming the contrary, i.e. $y_{0}<x_{1}^{*}\left(\xi_{x_{1}}\right)$, we deduce

$$
h_{\xi_{x_{1}}}^{y_{0}} \neq h_{\xi_{x_{2}}}^{y_{0}}
$$

Define

$$
x_{0}=\underset{1 \leqq i \leqq q}{\min }\left[y_{0} W_{i}^{-1}\left(\xi_{x_{1}}\right)\right] \leqq y_{0}
$$

Clearly, $y_{0}=x_{0}^{*}\left(\xi_{x_{1}}\right)$ and thus, since $y_{0}<x_{1}^{*}\left(\xi_{x_{1}}\right)$, we have $x_{0}<x_{1}$. Now, denoting by $N_{2}$ the set of all $1 \leqq i \leqq q$ for which

$$
x_{0}<y_{0} W_{i}^{-1}\left(\xi_{x_{1}}\right) \text {, i.e. } x_{0} W_{i}\left(\xi_{x_{1}}\right)<y_{0},
$$

and by $m \geqq 1$ the number of the remaining $i$ 's, we can easily calculate that $h_{\xi_{x_{1}}}^{y_{0}}$ and $h_{\xi_{x_{2}}}^{y_{0}}$ must satisfy the following equation for $h$

$$
\prod_{i=1}^{q} h_{\alpha_{i}}^{x_{0}\left[W_{1}\left(\xi_{x_{1}}\right) \xi_{x_{1}}\right]} \prod_{i \in N_{2}} h_{\xi_{x_{1}}}^{x_{0} W_{i}\left(\xi_{x_{1}}\right)} h^{m}=1,
$$

and must therefore be equal, in contradiction to (4.2). Hence

$$
x \xi_{x_{1}}=x \xi_{x_{2}} \text { for } x \in\left\langle 0, x_{1}^{*}\left(\xi_{x_{1}}\right)\right\rangle \text {. }
$$

Therefore, also $x_{1}^{*}\left(\xi_{x_{2}}\right)=x_{1}^{*}\left(\xi_{x_{1}}\right)$. As a consequence, if $x \in I$, then there is a unique $x \leqq x^{*} \leqq 1$ such that a function $\xi_{x}$ satisfying $(4.1)$ in $\langle 0, x\rangle$ is uniquely determined in $\left\langle 0, x^{*}\right\rangle$ and $x^{*}=x^{*}\left(\xi_{x}\right)$. In particular, this yields the uniqueness of the solution of (4.1) in $G_{H}$ provided that a solution exists. Moreover, if $x_{1}, x_{2} \in I$, then $x_{1}<x_{2}$ implies $x_{1}^{*}<x_{2}^{*}$ and, for any $x<x_{1}^{*}$, there is a unique $w$ such that

Now, put

$$
w \in I \text { and } w^{*}=x .
$$

$$
s=\sup _{x \in I} x \text { and } s^{\prime}=\sup _{x \in I} x^{*}
$$


Clearly, $0<s \leqq s^{\prime} \leqq 1$. We want to show $s=s^{\prime}=1$.

Using an indirect argument again, suppose that $s<1$. Define the function $\Psi_{2} \in G_{H}$ as follows: First of all, for $x \in\left\langle 0, s^{\prime}\right)$,

and

$$
x \Psi_{2}=x \xi_{w} \text {, where } w^{*}=x
$$

$$
s^{\prime} \Psi_{2}=\lim _{x \rightarrow s_{-}^{\prime}} x \Psi_{2}^{\prime} .
$$

Since $\Psi_{2}$ (for the time being defined in $\left\langle 0, s^{\prime}\right\rangle$ only) satisfies the equation $(4.1)$ in $\langle 0, s)$ and is uniquely determined in $\left\langle 0, s^{\prime}\right)$, we deduce, in view of continuity, that $\Psi_{2}$ satisfies $(4.1)$ in $\langle 0, s\rangle$ and is uniquely determined in $\left\langle 0, s^{*}\right\rangle$, where $s^{*}=s^{\prime}$. Also, since $s<1$, necessarily $s^{*} \Psi_{\mathbf{2}}<1$ and we can extend $\Psi_{2}$ on $\langle 0,1\rangle$ in such a way - by calculating the gradient $h_{\Psi_{2}}^{s^{*}}$ from a relation similar to $(4.3)$ - that $\Psi_{2}$ satisfies (4.1) in an interval $\left\langle 0, s+e_{2}\right\rangle$ with a suitable $e_{2}>0$. Hence, $s+e_{2} \in I-$ a contradiction of (4.4); consequently, $s=s^{*}=1$, as required.

Thus, there exists a solution $\xi_{1} \in G_{H *}$ of $(4.1)$ and - as shown above it is unique. Moreover, one can see immediately that

$$
\min _{\alpha_{i} \neq \varepsilon}\left(a_{\alpha_{1} 1}\right) \leqq a_{\xi_{1} 1} \text { and } a_{\xi_{1} \tau_{\xi_{1}}} \leqq \max _{\alpha_{i} \neq \varepsilon}\left(a_{\alpha_{i} \tau_{\alpha_{i}}}\right) \text {. }
$$

Hence, $\xi_{1}$ belongs to $G_{H}$.

The proof of Theorem 4.4 is completed.

Corollary 4.5. For every divisible $H \leqq R, G_{H}$ is an (algebraically) simple divisible (linearly) ordered group; in particular, $G_{H_{r}}$ is an (algebraically) simple divisible group admitting only two linear orders.

Theorem 4.6. Let $\varepsilon \neq \alpha$ be an element of $G_{H}$; let $\left\langle 0, a_{\alpha \mathbf{1}}\right\rangle,\left\langle a_{\alpha \tau_{\alpha}}, \mathbf{1}\right\rangle$ and $\left\langle a_{m}, b_{m}\right\rangle, m \in h$, be all non-trivial disjoint closed intervals complementary to the support of $x$; let $1 \leqq n_{0} \leqq \mathbf{N}_{0}$ be the number of the supporting intervals of $\alpha$. Then, the centre $Z_{\alpha}$ of the centraliser $C(\alpha)$ of $\alpha$ in $G_{H}$ is isomorphic to a direct product of $n_{0}$ copies of $H$ and

$$
C(\alpha) / Z_{\alpha} \cong G_{1} \times \prod_{m \in M} \vec{G}_{m} \times G_{2}
$$

where $G_{1} \cong G_{H *}, G_{2} \cong G_{* H}$ and $\bar{G}_{m} \cong \bar{G}_{H}$ for all $m \in M$.

Proof. By Lemma 3.6 and Proposition 3.7, every supporting interval of $\beta \in C(\alpha)$ is either a supporting interval of $\alpha-$ and $\beta$ is then in that supporting interval a (real) power of $\alpha$, or does not intersect the support of $\alpha$. On the other hand, any element whose support does not intersect the support of $\alpha$ belongs evidently to $C(\alpha)$. Thus, define

$\omega \in G_{1}$ if and only if $a_{\omega \tau_{\omega}} \leqq a_{\alpha 1}$, $\omega \in G_{m}$ if and only if $a_{m} \leqq a_{\omega 1}<a_{\omega \tau_{\omega}} \leqq b_{m}, m \in M$, $\omega \in G_{2}$ if and only if $a_{\alpha \tau_{\alpha}} \leqq a_{\omega 1}$, 
and Theorem $\mathbf{4 . 6}$ follows.

We conclude the paper with the following two simple remarks.

REMARK 4.7. (cf. [6]). With respect to its "natural" order (i.e. the order corresponding to the natural order of $R$ ), the group $G_{R}$ contains the continuum of non-order-isomorphic linearly ordered free subgroups of rank 2.

This is an immediate consequence of Lemma 3.8: Let

$$
0<a_{0}<b_{0}<a_{1}<a_{2}<\cdots<a_{n}<\cdots<1
$$

be an arbitrary sequence and $W_{n}(\xi, \eta)$ - the set of all "words" in $\xi, \eta$. For every $n \geqq 1$, let $\alpha_{n}$ and $\beta_{n}$ be two elements of $G_{R}$ whose supports are $I_{n}=$ $\left(a_{n}, a_{n+1}\right)$ and for which $W_{n}\left(\alpha_{n}, \beta_{n}\right) \neq \varepsilon$. Moreover, let $\alpha_{0}$ be a (simple) element of $G_{R}$ with the supporting interval $I_{0}=\left(a_{0}, b_{0}\right)$ and

$$
\beta_{0}=\alpha_{0}^{r} \text { for a real number } r \in R \text {. }
$$

Now, denote by $F(\alpha, \beta)$ the (free) subgroup of $G_{R}$ generated by $\alpha$ and $\beta$ defined by

and

$$
x \alpha=x \alpha_{n}, \quad x \beta=x \beta_{n} \quad \text { for } \quad x \in I_{n}, \quad n \geqq 0
$$

$$
x \alpha=x \beta=x \text { otherwise. }
$$

Evidently, the number $r \in R$ in (4.5) can be chosen in continuum different ways so that the resulting linearly ordered subgroups $F(\alpha, \beta)$ are nonorder-isomorphic.

It is apparent from the proof that there is an infinite number of nonorder isomorphic linearly ordered free subgroups of rank 2 in $G_{H}$ for every $H \subseteq R$. Also, let us point out here the trivial fact that $G_{R}$ (with its "natural" order) contains an isomorphic copy of any abelian linearly ordered group of countable "Archimedean rank" (cf. [8]).

REMARK 4.8. Let $H \subseteq R$ and $0<a<1$. Then the subgroup $G_{H}^{a} \subseteq G_{H}$ consisting of all $\alpha \in G_{H}$ such that $a x=a$ is a maximal subgroup of $G_{H}$.

We offer here a proof which retains its validity for every "transitive" subgroup of automorphisms of $(0,1)$.

Let $\beta$ be an arbitrary element of $G_{H} \backslash G_{H}^{a}$; without loss of generality, assume that $a \beta^{-1}>a$. In order to prove maximality of $G_{H}^{a}$ in $G_{H}$ it is obviously sufficient to show that any $\omega \in G_{H}$ such that $a \omega^{-1}>a$ belongs to the group generated by $G_{H}^{a}$ and $\beta$. Take

$$
0<\max \left(a \beta^{-1}, a \omega^{-1}\right)<b<1
$$

and denote by $\alpha$ a piecewise $H$-linear automorphism of $\langle 0, \mathbf{1}\rangle$ satisfying

and

$$
\left(a \omega^{-1}\right) \chi=a \beta^{-1}
$$


Now, since

$$
x \alpha=x \text { in }\langle 0, a\rangle \text { and }\langle b, 1\rangle \text {. }
$$

$$
a\left[(\alpha \beta)^{-1} \omega\right]=a \beta^{-1} \alpha^{-1} \omega=a,
$$

$\gamma=(\alpha \beta)^{-1} \omega$ belongs to $G_{H}^{a}$ and thus

as required.

$$
\omega=\alpha \beta \gamma \text { with } \alpha \text { and } \gamma \text { from } G_{H}^{a},
$$

\section{References}

[1] C. G. Chehata, 'An algebraically simple ordered group', Proc. London Math. Soc. 2 (1952), 183-197.

[2] C. J. Everett and S. Ulam, 'On ordered groups', Trans. Amer. Math. Soc. 57 (1945), 208-216.

[3] L. Fuchs, Partially ordered algebraic systems (Pergamon Press, Oxford-London-New York-Paris, 1963).

[4] G. Higman, 'On infinite simple permutation groups', Publ. Math. Debrecen 3 (1954), 221-226.

[5] Ch. Holland, 'The lattice-ordered group of automorphisms of an ordered set', Michigan Math. J. 10 (1963), 399-408.

[6] S. Matsushita, 'Sur la puissance des ordres dans un groupe libre', Proc. Koninkl. Nederl. Akad. Wet. A, 56 (1953), 15-16.

[7] J. Schreier and S. Ulam, 'Eine Bemerkung über die Gruppe der topologischen Abbildungen der Kreislinie auf sich selbst', Studia Math. 5 (1935), 155-159.

[8] H. H. Teh, 'Construction of orders in abelian groups', Proc. Cambridge Philos. Soc. 57 (1961), $476-482$.

Department of Mathematics

Institute of Advanced Studies

Australian National University

Canberra, A.C.T. 